\title{
Registration-Free Laparoscope Augmentation for Intra-Operative Liver Resection Planning
}

\author{
Marco Feuerstein ${ }^{a}$, Thomas Mussack ${ }^{b}$, Sandro M. Heining ${ }^{b}$, and Nassir Navab ${ }^{a}$ \\ ${ }^{a}$ Chair for Computer Aided Medical Procedures (CAMP), Technische Universität München, \\ Boltzmannstr. 3, 85748 Garching, Germany; \\ ${ }^{b}$ Chirurgische Klinik und Poliklinik, Klinikum der LMU - Innenstadt, Nußbaumstraße 20, \\ 80336 München, Germany
}

\begin{abstract}
In recent years, an increasing number of liver tumor indications were treated by minimally invasive laparoscopic resection. Besides the restricted view, a major issue in laparoscopic liver resection is the enhanced visualization of (hidden) vessels, which supply the tumorous liver segment and thus need to be divided prior to the resection. To navigate the surgeon to these vessels, pre-operative abdominal imaging data can hardly be used due to intraoperative organ deformations mainly caused by appliance of carbon dioxide pneumoperitoneum and respiratory motion. While regular respiratory motion can be gated and synchronized intra-operatively, motion caused by pneumoperitoneum is individual for every patient and difficult to estimate.

Therefore, we propose to use an optically tracked mobile C-arm providing cone-beam CT imaging capability intraoperatively. The $\mathrm{C}$-arm is able to visualize soft tissue by means of its new flat panel detector and is calibrated offline to relate its current position and orientation to the coordinate system of a reconstructed volume. Also the laparoscope is optically tracked and calibrated offline, so both laparoscope and C-arm are registered in the same tracking coordinate system.

Intra-operatively, after patient positioning, port placement, and carbon dioxide insufflation, the liver vessels are contrasted and scanned during patient exhalation. Immediately, a three-dimensional volume is reconstructed. Without any further need for patient registration, the volume can be directly augmented on the live laparoscope video, visualizing the contrasted vessels. This augmentation provides the surgeon with advanced visual aid for the localization of veins, arteries, and bile ducts to be divided or sealed.
\end{abstract}

Keywords: Enhanced Reality, Image-Guided Therapy, Calibration, Abdominal Procedures, Visualization

\section{INTRODUCTION}

The liver is one of the most frequent target organs for primary and secondary malignant lesions. Hepatocellular carcinoma (HCC) and metastasis of colorectal neoplasms are the most common diagnoses. HCC with about one million newly registered cases worldwide each year is the fourth most frequent cancer related cause of death. Without medical treatment, HCC will lead to death in $100 \%$ of the cases. At present, only about 5-15\% of diagnosed cases can be treated by the surgical resection of malignant regions, which is standard of care. Ablation and chemotherapy do not provide a sufficient success rate. The average five year survival rate only lies between 30 and $40 \% .^{1}$

\footnotetext{
Further author information: (Send correspondence to M.F.)

M.F.: $\quad$ E-mail: feuerste@cs.tum.edu

T.M.: E-mail: thomas.mussack@med.uni-muenchen.de

S.M.H.: E-mail: sandro-michael.heining@med.uni-muenchen.de

N.N.: $\quad$ E-mail: navab@cs.tum.edu
}

Copyright 2007 Society of Photo-Optical Instrumentation Engineers.

This paper was published in SPIE Medical Imaging and is made available as an electronic reprint with permission of SPIE. One print or electronic copy may be made for personal use only. Systematic or multiple reproduction, distribution to multiple locations via electronic or other means, duplication of any material in this paper for a fee or for commercial purposes, or modification of the content of the paper are prohibited. 
Since 1991, laparoscopic liver resection is performed for an increasing number of cases, as it is less invasive than open surgery (laparotomy) and, hence, less harmful to the patient. However, laparoscopic surgery itself introduces several drawbacks at the same time. The localization of the target region, i.e. the vessels to be sealed or clipped, is difficult to achieve due to a restricted working space and a visualization solely limited to the view of the laparoscope onto the liver surface. Additionally, up to $14 \%$ of interventions need to be converted to laparotomy because of intra-operative bleeding. ${ }^{2}$ Here a selective minimally invasive therapy enabled by improved intra-operative imaging as well as a robust and accurate navigation will be of great benefit.

In a typical totally laparoscopic liver resection procedure, a major step towards tumor resection is the successful division of all vessels supplying the respective liver segment. For laparoscopic abdominal surgery, the target region can be deformed due to heartbeat and respiratory motion. While deformations in the abdominal area caused by the heartbeat are negligible, ${ }^{3}$ the rather large respiratory motion of about $1 \mathrm{~cm}$ can be corrected for by gating ${ }^{4,5}$ and can be synchronized to e.g. an augmented visualization. Individual deformations of greater extent however occur between pre-operative acquisition of the CT and the beginning of the resection, i.e. during patient and port placement, appliance of carbon dioxide pneumoperitoneum, and the intervention itself. Pneumoperitoneum alone can already cause large liver motions of e.g. $1.8 \pm 12,4.1 \pm 6.4$, and $0.1 \pm 0.4 \mathrm{~mm}$ in x, $\mathrm{y}$, and z directions, respectively, as shown for two pigs. ${ }^{4}$ In this case, using pre-operative rigid imaging data requires an additional intra-operative registration process, which is costly in terms of time and interaction. Therefore, precisely guiding the surgeon to the target area is difficult to perform and hard to validate.

For orthopedics and neurosurgery, where mainly rigid structures are involved, navigation systems aligning pre-operative imaging data in respect to the patient are commercially available*. For laparoscopic abdominal surgery, the target region is deformed in a great extent during patient and port placement, appliance of $\mathrm{CO}_{2}$ pneumoperitoneum, respiration, and the intervention itself. In that case, using pre-operative rigid imaging data to accurately guide the surgeon to the target area is difficult to perform and hard to validate. Instead, initial attempts were and need to be made to use intra-operative imaging. In general, MRI scanners are too bulky to be used during laparoscopic surgery or require the patient to be moved for the acquisition, making a precise intra-operative registration almost impossible. Fichtinger et al developed an intra-operative CT image overlay system for needle insertion, where no major deformations are involved. ${ }^{6}$ Showing only a single CT slice, it would be difficult to use their system for laparoscopic vessel augmentation, where volumetric 3D data is essential.

To guide the division of vessels and the resection, ultrasonography may be used. It is however difficult to understand, how ultrasound (US) images are oriented in relation to the patient. Ellsmere et al therefore propose an advanced system to intuitively display the laparoscopic US image plane relative to a pre-operative 3D model of the patient. ${ }^{7}$ This helps the physician to identify anatomical key structures and to learn the use of laparoscopic ultrasound. An ideal complement to their navigation system would be the provision of registered intra-operative $3 \mathrm{D}$ data of high quality, which is not affected by the individual organ movement between pre-operative data acquisition and the beginning of the intervention (after $\mathrm{CO}_{2}$ insufflation) and could be used instead of the preoperative CT to accomplish the guidance. Another approach to improve the spatial relation of US images to the patient is taken by Leven et al. ${ }^{8}$ They propose a system to apprehensively overlay the laparoscopic ultrasound image plane or a reconstructed US volume, respectively, directly on the live images of a stereo endoscope. It is however still difficult to interpret low-resolution US images, especially reconstructed volumes. Providing a high-resolution 3D volume intra-operatively and combining it with advanced visualization could be an optimal supplement to their system as well.

Supplementary to laparoscopic ultrasound, we introduce the use of a mobile isocentric C-arm providing conebeam CT imaging capability ${ }^{9}$ to visualize soft tissue intra-operatively, which is not possible with current commercially available mobile C-arms. An optical tracking system determines the position and orientation of both $\mathrm{C}$-arm and laparoscope, which can be brought into the same coordinate system by various offline calibration routines, as described in section 2.2. Intra-operatively, after port placement, appliance of $\mathrm{CO}_{2}$ pneumoperitoneum, and instrument insertion, we contrast the vessel tree of the liver and at the same time acquire a C-arm image series during patient exhalation. Alternatively, C-arm projections can be gated and correlated to respiratory motion in order to acquire a high-quality scan, as Kriminski et al suggested. ${ }^{10}$ After reconstruction, we are able to precicely augment the contrasted vessel tree directly on the laparoscopic view just before the beginning of

\footnotetext{
*e.g. by Aesculap, BrainLAB, Medtronic, ORTHOsoft, PI Systems, Praxim Medivision, and Stryker
} 
the resection without any time-consuming patient registration process. The augmentation can be synchronized to the patient's respiration and only be displayed during exhalation. ${ }^{3}$ This provides the surgeon with valuable information on the location of veins, arteries, and bile ducts, which supply the liver segment to be resected and therefore need to be divided.

\section{SYSTEM OVERVIEW}

Figure 1 shows the system components we used to conduct all calibration steps, experiments, and ex vivo studies.

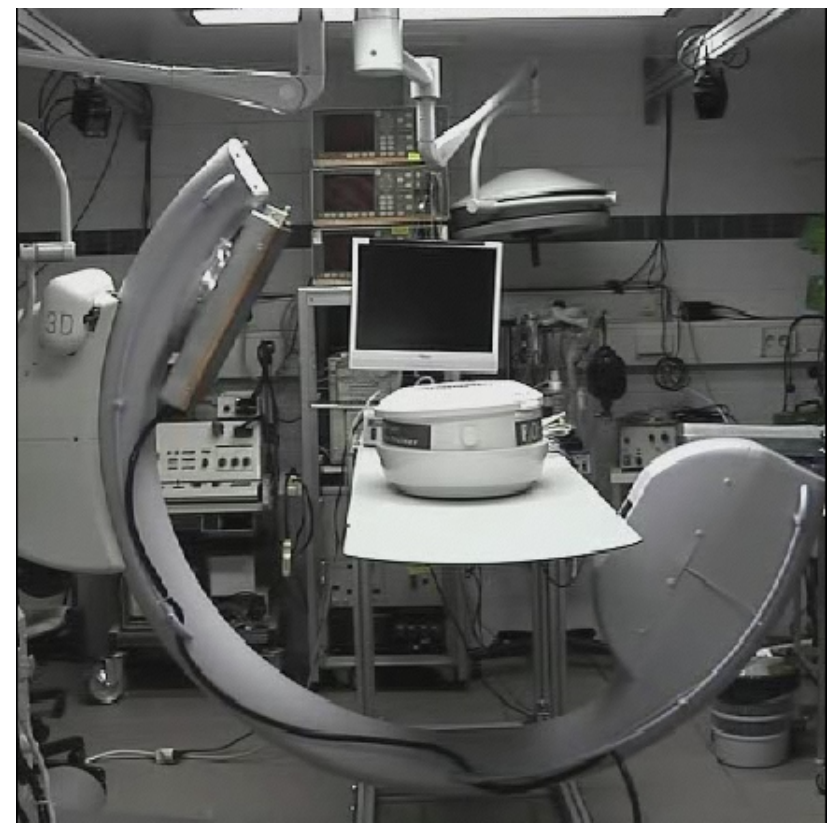

(a) Arrangement of our experimental setup in the animal operating room.

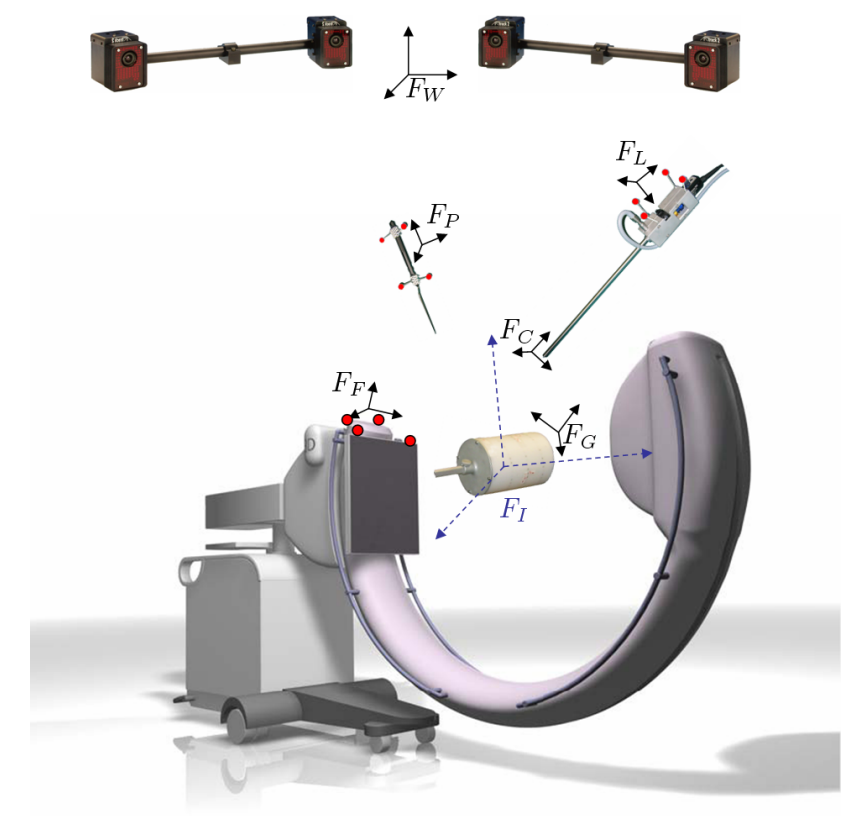

(b) All involved coordinate frames, which need to be brought into a common world coordinate system.

Figure 1. System overview.

\subsection{System Setup}

The prototype mobile C-arm is based on a Siemens PowerMobil and incorporates a workstation deployed with acquisition and reconstruction software by Siemens. We attached four retroreflective spherical markers to the flat-panel detector of the C-arm. These markers are seen by four optical tracking cameras (ARTtrack2, ART $\mathrm{GmbH}$ ) mounted just beneath the operating room's ceiling, one in each corner of a rectangle. The tracking system is connected to a PC equipped with a tracking software that is able to send $6 \mathrm{D}$ tracking data over Ethernet to our navigation workstation. This navigation workstation is also connected to the C-arm workstation via Ethernet to directly access any reconstructed volumes. A frame grabber is incorporated that captures the live video of our laparoscope with $30^{\circ}$ optics (KARL STORZ GmbH \& Co. KG). Four retroreflective spherical markers are each attached to the laparoscope's body and a pointer. We utilize a helical BB phantom for geometric $\mathrm{C}$-arm calibration. The operating table is made of carbon to get as little imaging artifacts as possible during an acquisition.

\subsection{System Calibration}

In order to augment C-arm data on the laparoscopic view, all involved coordinate systems need to be brought into one common world coordinate system (= tracking coordinate system). Therefore, two main transformations in space need to be computed: 1) The transformation from the markers attached to the laparoscope's body to the camera center along with the projection geometry of the laparoscope camera. 2) The transformation from 
the markers attached to the flat-panel detector to the isocenter of the C-arm. All calibration steps can be done off-line. Their results are valid for a long period of time and only need to be repeated once in a while.

Laparoscope Calibration We use a planar checkerboard pattern with squares of $10 \mathrm{~mm}$ unit length to calibrate the laparoscope. As described in our prior work, ${ }^{11}$ the pattern is viewed from several arbitrary poses satisfying distinguishable rotation axes. So the intrinsic camera parameters and distortion coefficients can be determined from several 2D-3D point correspondences. Additionally, the hand-eye formulation $A X=X B$ can be solved ${ }^{12}$ to compute the missing static transformation from the markers on the laparoscope's body to the camera center $\left({ }^{C} T_{L}\right)$.

C-Arm Calibration We adopted the C-arm calibration method proposed in ${ }^{13}$ to the new C-arm prototype and our tracking system. First, a geometric C-arm calibration is performed using a helical pattern of BBs in a cylindrical, hollow phantom, where the coordinates of all BBs' centroids are exactly known in the phantom coordinate system. This geometric calibration determines the projection geometry for each of the poses during a $180^{\circ}$ rotation of the $\mathrm{C}$-arm. Knowing the projection geometry, the coordinates of the BBs can be transformed from the geometric calibration phantom to a coordinate system, whose center coincides with the C-arm's isocenter $\left({ }^{I} T_{G}\right)$.

For a tracked pointer we performed a tip (hot spot) calibration by pivoting the pointer around a fixed tip location. This pointer is used to acquire the 3D coordinates of the BBs' outward surface on the phantom in the tracking coordinate system. To compensate for the fact that the pointer only touches the surface of a BB and not its centroid, an offset needs to be computed for each BB. This offset equals a BB's radius and is along the orthogonal to the tangent touching the surface of the phantom.

By matching the coordinates of the point set acquired with the pointer and the corresponding BBs' coordinates in the calibration phantom, we can determine the transformation from the tracking to the geometric calibration phantom's coordinate system $\left({ }^{G} T_{W}\right)$. The transformation from the flat-panel markers to the tracking system is directly measured by the tracking system $\left({ }^{W} T_{F}\right)$. The final transformation ${ }^{I} T_{F}$ from the flat-panel markers to the isocenter can now be computed by ${ }^{I} T_{G}{ }^{G} T_{W}{ }^{W} T_{F}$. This calibration is done with the C-arm in its start position, which is the only requirement for obtaining a valid ${ }^{I} T_{F}$ intra-operatively. During an intervention, the transformation ${ }^{I} T_{W}={ }^{I} T_{F}{ }^{F} T_{W}$ can be computed once, so the C-arm can be removed after the acquisition.

\subsection{Visualization}

Our navigation workstation is equipped with 2 GB main memory, an Athlon $643500+$ CPU, and an NVIDIA GeForce 6800 Ultra graphics card with $256 \mathrm{MB}$ memory. The reconstruction software of the C-arm generates a 16 bit gray level volume of $512 \times 512 \times 384$ voxels. Using the CG shader language, we are able to directly render this volume in real time as view aligned 3D textures. We augment the volume rendering directly on the live laparoscope video that is also undistorted at the same time. To visualize certain tissues only, specific colors and opacities can be interactively assigned to the rendered textures, provided by a graphical transfer function editor. Alternatively, default transfer functions can be loaded, for instance tailored to contrasted vessels. To easily synchronize tracking with video data and visualize the augmentation, we use our medical augmented reality framework CAMPAR. ${ }^{14}$ In general, the augmented visualization will only be shown to the surgeon for the intra-operative in-situ planning of the resection to provide a detailed 'roadmap' of the vessels, but not any more when the surgeon starts to cut, since this causes the liver to deform again and invalidates any prior registration.

\section{ACCURACY EVALUATION}

To evaluate the overall system accuracy, we performed two types of experiments. First the navigation accuracy with the tracked pointer was assessed, second the augmentation accuracy using the tracked laparoscope. 
Navigation Error To determine the overall system's navigation accuracy, we used the cubic evaluation phantom similar to the one proposed by Ritter et al that contains cylindrical bore holes of varying depth. ${ }^{13}$ The phantom was adhered to the operating table with double-sided adhesive tape, so it could not be moved any more. Then the varying depth of 20 bore holes was measured in terms of the pointer tip's 3D position in the tracking coordinate system. The tip coordinates were transformed into the volume coordinate system by ${ }^{I} T_{F}{ }^{W} T_{F}{ }^{-1}$. Now the same 20 holes were filled with BBs of radius $r=1.5 \mathrm{~mm}$. Afterwards, a volume was reconstructed, wherein the BBs' centroids were extracted automatically by a hybrid segmentation algorithm based on thresholding, region growing, the BBs' shape, and weighing of voxel intensities.

Since the bore holes have an inclination $\alpha$ of $56^{\circ}$, the distance from a BB's centroid to the end of the bore hole equals $r / \cos \alpha$. This distance offset needs to be applied to all segmented centroids to be theoretically aligned with the tip of the pointer.

The overall Euclidean root mean square (rms) error between the measured and segmented coordinates of the BBs' centroids was $1.10 \mathrm{~mm}$, which confirms previous results of Ritter et al. ${ }^{13}$ A second experiment after a complete system recalibration using BBs of $1.0 \mathrm{~mm}$ radius resulted in a reproducible Euclidean rms error of 1.05 $\mathrm{mm}$.

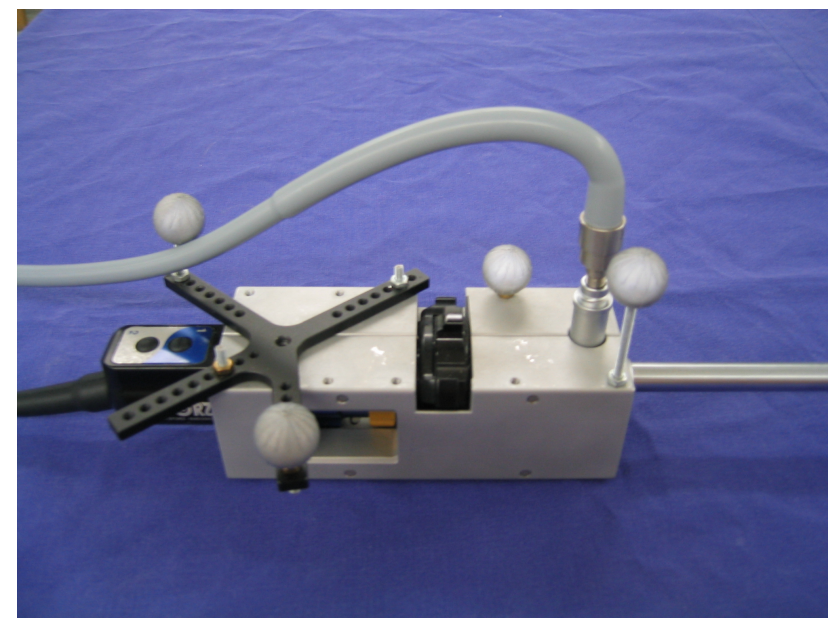

(a) Initial sub-optimal marker configuration.

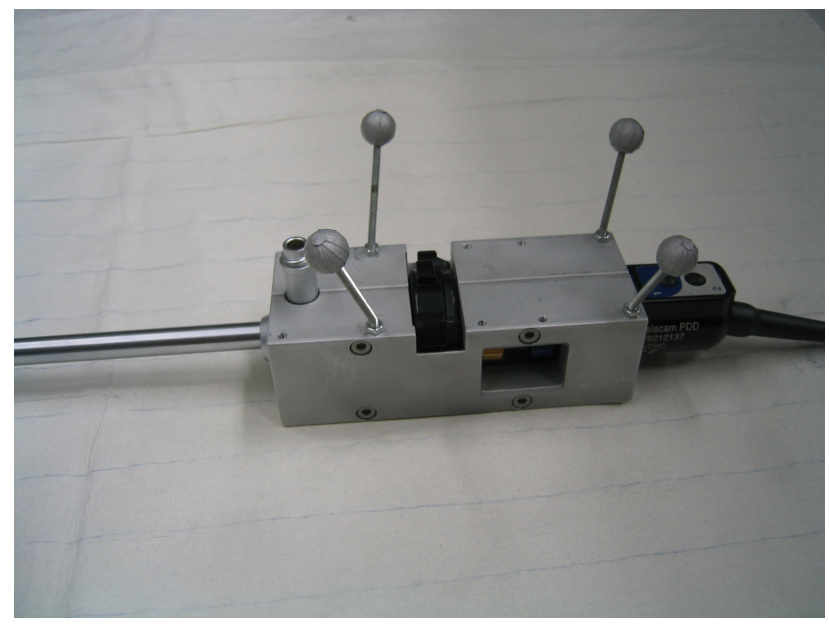

(b) Rearranged markers to ensure optimal tracking properties.

Figure 2. Two alternative marker target configurations.

Augmentation Error For the determination of the laparoscopic augmentation error, a volume of a plastic model of a heart with three adhered spherical markers of $2.3 \mathrm{~mm}$ diameter and a curved line marker (CTSPOTS ${ }^{\circledR}$, Beekley Corporation) representing a vessel was reconstructed. The laparoscope was moved around this plastic model arbitrarily. Augmenting the live laparoscope video, images were taken from a large number of views covering the interventional working space. On all images the markers and their augmentated counterparts, visualized in a different color, are visible (cf. figure 3). The absolute distance in $\mathrm{mm}$ was measured from the midpoint of a spherical marker and its corresponding virtual projection. The obtained rms error was $1.78 \mathrm{~mm}$ and the maximum error $4.14 \mathrm{~mm}$. This high error was caused by an inappropriate configuration of the markers on our laparoscope, leading to unstable tracking, e.g. by partial or complete occlusions of the markers. This behaviour could be anticipated, since the calibrated camera center is located about $350 \mathrm{~mm}$ away from the markers, leading to a large extrapolation displacement for small angular or translational tracking errors of the makers.

Therefore, in a second experiment we rearranged the four markers on the laparoscope following the heuristics proposed by West et al ${ }^{15}$ (cf. figure 2), so they could be seen optimally by the tracking system in almost every possible position. After a recalibration of the laparoscope, we were able to significantly improve the augmentation accuracy from all positions of the laparoscope and could decrease the rms error to only $0.81 \mathrm{~mm}$ and the maximum 
error to $1.38 \mathrm{~mm}$.

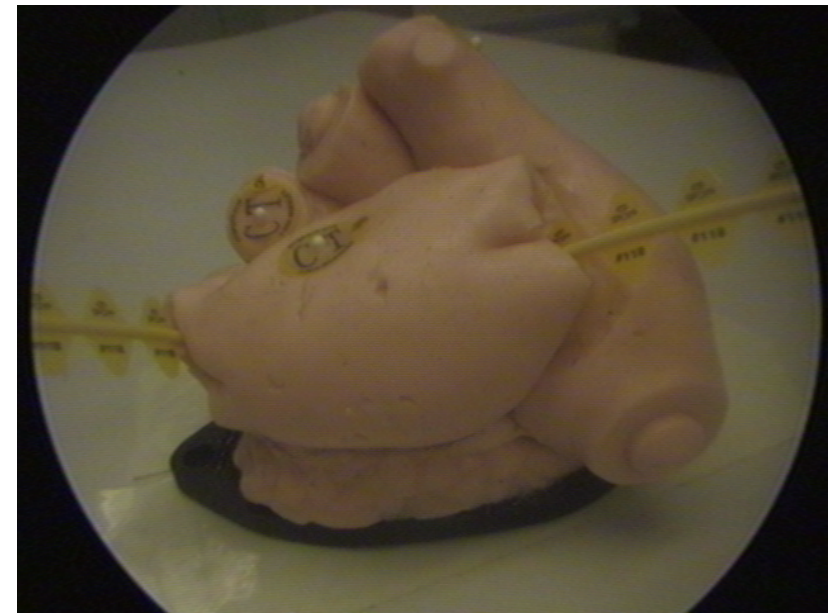

(a) Real, undistorted laparoscope image showing the 3 spherical markers and a curved line marker on the plastic heart.

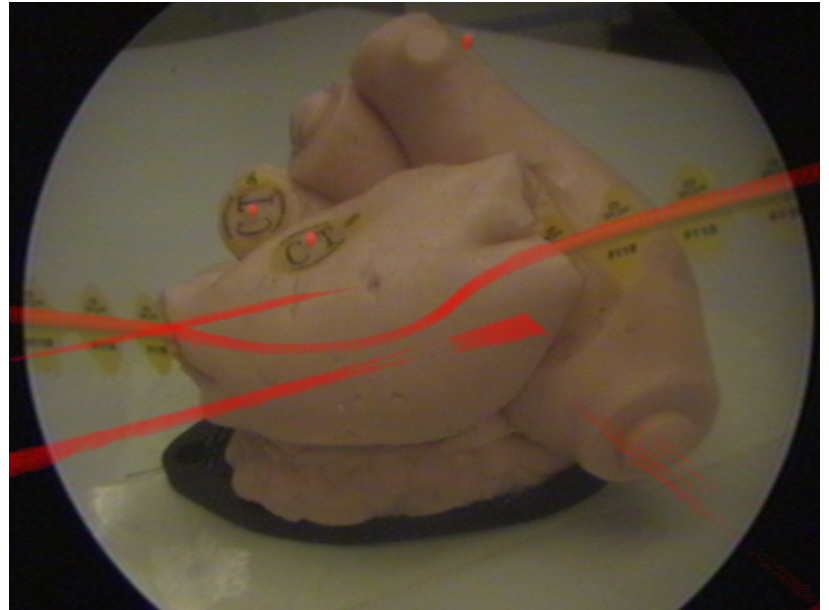

(b) Augmented laparoscope image. Volume rendered are only the markers; the additional lines are artifacts in the reconstructed volume.

Figure 3. Plastic heart used for the determination of the augmentation error.

\section{EX VIVO PERFUSION STUDIES}

To clinically evaluate our vessel augmentation, we performed two ex-vivo studies together with our clinical partners, one with a freshly harvested whole porcine liver and a second one with a defrosted whole ovine kidney. Each time we placed the organ in a perfusion box (Pulsating Organ Perfusion Trainer, OPTIMIST Handelsges.m.b.H.).

The surgeons catheterized the liver's portal vein and the kidney's renal artery, respectively. Then the iodinated nonionic contrast agent SOLUTRAST ${ }^{\circledR} 300$ was administered into the organ. It was diluted in normal saline and conveyed into the organ by the pump of the perfusion system. We immediately started a C-arm acquisition and reconstructed the organ's 3D volume. In another experiment, we directly injected the pure contrast agent.

The acquisition of $200 \mathrm{C}$-arm projections takes 64 seconds, which stays within the time limits of holding breath during artificial respiration. After acquisition, the reconstruction of the 3D volume was started, which currently takes about six minutes. However, once the prototype system is commercialized, the reconstruction algorithms will be optimized and parallelized. Additionally, reconstruction will commence as soon as the first projection is acquired. This will lead to reconstruction times of approximately one minute after completion of a scan, as known from modern CT scanners.

The contrasted part was clearly visible in the reconstructed volume for both cases, directly as well as via the perfusion system. The vessels were directly augmented on the laparoscope's view by volume rendering. This direct visualization technique does not require any processing time, since time-consuming segmentation, as it would be needed for surface-based rendering, can be completely avoided. The vessels could be accurately overlaid for most laparoscope positions, as you can see in figure 4(a) in the case of the kidney, where the real and virtual catheters perfectly overlap. In the case of the porcine liver, only the vessels of one lobe could be contrasted partially, as some liver vessels were accidentally cut at the abattoir.

Although in general more vessels could be visualized for the direct administration of contrast agent, artifacts appeared during the $3 \mathrm{D}$ volume reconstruction due to the high concentration. We will conduct further studies to find an optimal contrast agent concentration for an artifact-free reconstruction and a clear visualization of all relevant vessels. 


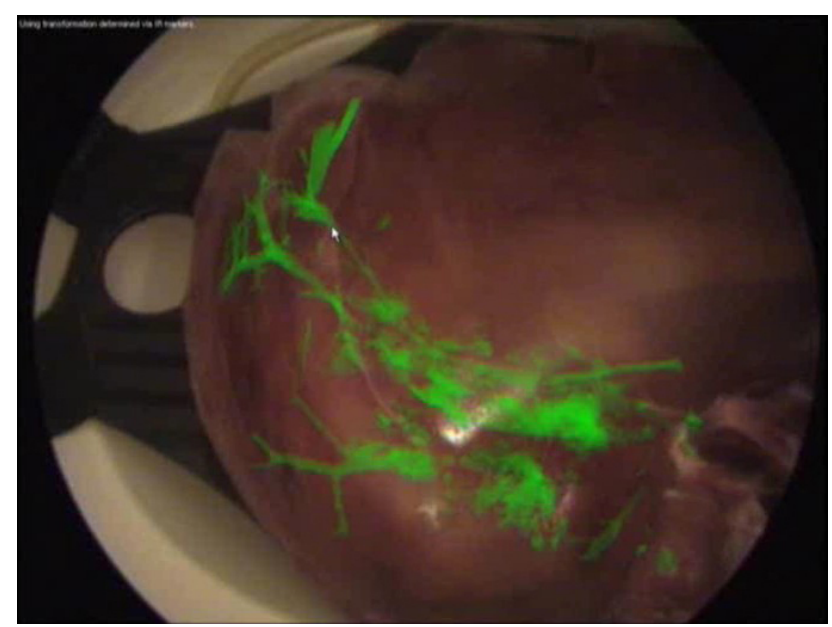

(a) Augmented porcine liver. The speckled areas are reconstruction artifacts caused by a high concentration of contrast agent.

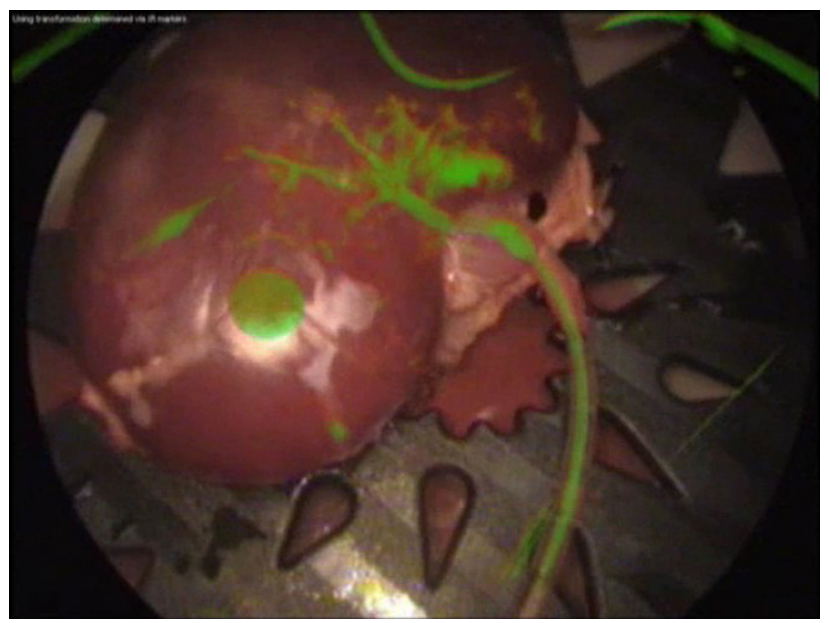

(b) Augmented ovine kidney. The big spot is the perfusion system's plug, which apparently has a similar Hounsfield unit like the contrast agent.

Figure 4. Vessel augmentation.

\section{CONCLUSION}

This work is an important and encouraging step towards the provision of precise intra-operative visualization aid for laparoscopic liver resection providing automatic intra-operative patient registration. Combining intraoperative imaging, gating, tracking, and visualization, restrictions caused by organ movement between pre- and intra-operative imaging can be easily overcome. Our intra-operative resection planning system is not solely restricted to liver surgery, but can also be applied to various other endoscopic interventions. Our experiments and studies show satisfying qualitative results for intra-operative laparoscope augmentation.

The result provided here could allow different methods proposed in the literature ${ }^{7,8}$ to start with co-registered intra-operative cone beam reconstruction in order to improve their advanced visualization solutions.

\section{ACKNOWLEDGMENTS}

The authors would like to thank Siemens Medical for the loan of the prototype C-arm system, especially Dr. Rainer Graumann and Christian Schmidgunst for their support. Also the loan of an entire laparoscope system by KARL STORZ GmbH \& Co. KG is greatly appreciated. The authors are also grateful to Dr. Peter Scheuber for the provision of the experimental operating room and various equipment. Finally, this work might not have been possible had it not been for the efforts of Martin Horn, Christoph Bichlmeier, and Andreas Keil, who greatly supported the authors during the experiments.

\section{REFERENCES}

1. M. B. Thomas and A. X. Zhu, "Hepatocellular carcinoma: The need for progress," Journal of Clinical Oncology 23(13), pp. 2892-2899, 2005.

2. T. Mala and B. Edwin, "Role and limitations of laparoscopic liver resection of colorectal metastases," Digestive Diseases 23, pp. 142-150, 2005.

3. B. Olbrich, J. Traub, S. Wiesner, A. Wiechert, H. Feußner, and N. Navab, "Respiratory motion analysis: Towards gated augmentation of the liver," in Proceedings of Computer Assisted Radiology and Surgery (CARS 2005), pp. 248-253, (Berlin, Germany), June 2005.

4. A. Herline, J. Stefansic, J. Debelak, S. Hartmann, C. Pinson, R. Galloway, and W. Chapman, "Image-guided surgery: preliminary feasibility studies of frameless stereotactic liver surgery," Archives of Surgery 134, pp. 644-650, June 1999. 
5. M. Clifford, F. Banovac, E. Levy, and K. Cleary, "Assessment of hepatic motion secondary to respiration for computer assisted interventions," Computer Aided Surgery 7(5), pp. 291-299, 2002.

6. G. Fichtinger, A. Deguet, K. Masamune, E. Balogh, G. S. Fischer, H. Mathieu, R. H. Taylor, S. J. Zinreich, and L. M. Fayad, "Image overlay guidance for needle insertion in ct scanner," IEEE Transactions on Biomedical Engineering 52(8), pp. 1415-1424, 2005.

7. J. Ellsmere, J. Stoll, D. W. Rattner, D. Brooks, R. Kane, W. M. Wells, III, R. Kikinis, and K. Vosburgh, "A navigation system for augmenting laparoscopic ultrasound," in Proc. Int'l Conf. Medical Image Computing and Computer Assisted Intervention (MICCAI), pp. 184-191, 2003.

8. J. Leven, D. Burschka, R. Kumar, G. Zhang, S. Blumenkranz, X. D. Dai, M. Awad, G. D. Hager, M. Marohn, M. Choti, C. Hasser, and R. H. Taylor, "Davinci canvas: A telerobotic surgical system with integrated, robotassisted, laparoscopic ultrasound capability," in Proc. Int'l Conf. Medical Image Computing and Computer Assisted Intervention (MICCAI), Lecture Notes in Computer Science 3749, pp. 811-818, September 2005.

9. J. H. Siewerdsen, D. J. Moseley, S. Burch, S. K. Bisland, A. Bogaards, B. C. Wilson, and D. A. Jaffray, "Volume ct with a flat-panel detector on a mobile, isocentric c-arm: Pre-clinical investigation in guidance of minimally invasive surgery," Medical Physics 32, pp. 241-254, January 2005.

10. S. Kriminski, M. Mitschke, S. Sorensen, N. M. Wink, P. E. Chow, S. Tenn, and T. D. Solberg, "Respiratory correlated cone-beam computed tomography on an isocentric c-arm," Physics in Medicine and Biology 50, pp. 5263-5280, November 2005.

11. M. Feuerstein, S. M. Wildhirt, R. Bauernschmitt, and N. Navab, "Automatic patient registration for port placement in minimally invasive endoscopic surgery," in Medical Image Computing and Computer-Assisted Intervention - MICCAI 2005, 8th International Conference, J. S. Duncan and G. Gerig, eds., Lecture Notes in Computer Science 3750, pp. 287-294, Springer-Verlag, (Palm Springs, CA, USA), September 2005.

12. R. Tsai and R. Lenz, "Real time versatile robotics hand/eye calibration using 3d machine vision," in Proceedings of the IEEE International Conference on Robotics and Automation, 1, pp. 554-561, 1988.

13. D. Ritter, M. Mitschke, and R. Graumann, "Markerless navigation with the intra-operative imaging modality siremobil iso-c ${ }^{3 \mathrm{D}}$," electromedica 70(1), pp. 31-36, 2002.

14. T. Sielhorst, M. Feuerstein, J. Traub, O. Kutter, and N. Navab, "Campar: A software framework guaranteeing quality for medical augmented reality," International Journal of Computer Assisted Radiology and Surgery 1, pp. 29-30, June 2006.

15. J. West and C. Maurer, "Designing optically tracked instruments for image-guided surgery," IEEE Transactions on Medical Imaging 23, pp. 533-545, May 2004. 